\title{
A Novel Four Rotor Aircraft Adaptive Control Algorithm Based on the Optimized Fuzzy Neural Network
}

\author{
Yizhou Wei ${ }^{1}$, GangQiang Sun ${ }^{2}$, Shuheng Wang ${ }^{3}$ \\ ${ }^{1}$ Electric And Computer Engineering, UM-SJTU Joint Institute, Shanghai Jiaotong University, \\ 200240 \\ 2.Schooled at home, Rui'an Wenzhou, China \\ 3. University of California, San Diego, UCSD department of mathematics
}

San Diego, CA 92092

Keywords: four rotor aircraft adaptive control algorithm (4R2AC) algorithm, back-stepping (BS).

\begin{abstract}
A novel four rotor aircraft adaptive control algorithm, taking into account fluffy neural system, is described in this paper. Concentrating on the unverifiable streamlined coefficients which describe the push and torques, an adaptive controller for a four-rotor aircraft is composed in view of Immersion and Invariance approach. A moderately basic model parameterized concerning a couple of obscure parameters is created in order to manage the complex multivariable parameter estimation. For the estimation of those parameters, the four rotor aircraft adaptive control algorithm (4R2AC) algorithm is utilized. The control algorithm is based on the optimized fuzzy neural network method. Numerical recreation delineates the heartiness of the controller against the parametric vulnerabilities and outside aggravations.
\end{abstract}

\section{Introduction}

A quad-rotor aircraft is an under-impelled, firmly coupled nonlinear framework with parameter instability and un-demonstrated unsettling influence. Aiming at making the aircraft track the sought direction, a settled twofold circles control framework is described in this manuscript. A position blunder corresponding subsidiary controller is planned as the external circle controller taking into account the coupling activity amongst rotational and translational development, and an adaptive backstepping (BS) sliding mode control algorithm is utilized to settle the state of mind. The Quadruple-Rotors are recognized as a perfect stage for automated frameworks, because of its effortlessness, vertical flight and high mobility. The progression of Quadruple-Rotors are nonlinear and multivariate. Because of the nonlinear way of airborne vehicles, nonlinear control techniques appears to be better decisions for Quadruple-Rotor to handle nonlinearities and have more extensive execution. Among every one of these strategies, BS is a surely understood and intense control strategy widely utilized for nonlinear frameworks.

Flying items must adapt to various wellsprings of vulnerability in the framework model, outer unsettling influences and blames in estimations. The issue is far more severe in nearness of wind unsettling influence. Adaptive control is a proper contender for this kind of aircraft for its capability of producing superior tracking within the sight of wind unsettling influences and parametric vulnerabilities. In [1], an adaptive control algorithm taking care of the direction following issue for Quadruple-Rotor flying vehicles is inferred utilizing a back venturing system. In [2] another methodology for the demeanor control of a Quadruple-Rotor aircraft is proposed laying on the mix of the BS system and a nonlinear vigorous relative fundamental controller. Additionally in [3], adaptive following control of an under-actuated Quadruple-Rotor is tended to utilizing state criticism control framework and an integrator BS approach with a projection-based adaptive plan to assess the obscure parameters.

In this work, we address the issue of direction following for Quadruple-Rotor by utilizing adaptive BS control approach. The adaptive control algorithm is based on the optimized fuzzy neural network method. Configuration of the control law is done on the nonlinear model of Quadruple-Rotor, considering parametric vulnerabilities. 


\section{Related Works}

The history of quad-rotor aircraft starts from early 1900s, when two French engineer build the first quadrotor helicopter which has no control mechanism and therefore poor performance. Then, despite some tentative work in the 1920s and 1950s which suffered from poor stability, the progress on quadrotor aircraft is rather slow. It was not until recent that the development of senor technology and control theory inspired the evolution of micro quad-rotor aircrafts. Today, the application of quad-rotor helicopters can be seen everywhere, such as package delivery and aerial photo. Furthermore, with the help of self-adapting control algorithm, the quadrotor helicopter can be more easily applied to serious environment.

Aiming at making the aircraft track the coveted direction, in [4] a settled twofold circles control framework is received in this manuscript. A position blunder corresponding subordinate (PD) controller is composed as the external circle controller taking into account the coupling activity amongst rotational and translational development, and an adaptive BS sliding mode control algorithm is utilized to balance out the disposition. The work in [5] depicts the use of immediate and roundabout model reference adaptive control to a lightweight ease Quadruple-Rotor.

Albeit nonlinear controllers perform well in reproduction and trials, since they rely on upon exact framework, the continuous exhibitions of these control laws are worse than direct controllers. Therefore, because of the constrained installed registering assets of the Quadruple-Rotor, outlining a control law for even decisively state of mind adjustment, additionally has solid hostile to aggravation way. Fourfold Rotor unsettling influences can be utilized to communicate to exogenous inputs, for example, steady wind and model befuddled instabilities, for example, floats in measuring scopes and nonsymmetrical mass conveyance [6-7].

Recently proposed methodologies commonly depend on delicate registering procedures. Among these methods, Neural Network (in the blink of an eye NN) controllers have been proposed as adaptive controller for nonlinear framework. NN controllers speak to an extremely productive apparatus in flight control.

Recognizing the recent progress on NN, this paper describes an adaptive control algorithm based on the optimized fuzzy neural network. A point by point investigation is completed keeping in mind the goal to accept the utilization of NNs in flight control applications. The proposed control framework fulfills standard conditions for solidness, and demonstrates that adaptive Neural Network can achieve the floating arrangement even in nearness of disappointments and harm [8-10]. A security and merging examination of a NN adaptive flight control is also introduced. Considering the shakiness identified with high-pick up taking in, a recursive minimum squares learning law is introduced. Numerical results demonstrate that high recurrence motions, because of un-modeled flow, can be excluded by the introduced method.

The principle idea of the adaptive control algorithm described in this paper comprises in keeping away from mistake brought on by numerical displaying and actuator harms through adaptive NN. The variety of streamlined coefficients is proposed, in which on-line learning NNs, taking into account control hypothesis for weight overhaul standards, are executed. The proposed approach is by all accounts more steady than ordinary back-venturing controller. At long last, the adaptive $\mathrm{NN}$ technique introduced in proofs that a joint association amongst online and foundation learning strategies produces a strong and effective adaptive flight controller which ensures great execution even in questionable situations.

\section{Adaptive and non adaptive control stratergies}

It is expected that the Quadruple-Rotor is an unbending body. The elements of the QuadrupleRotor can be decayed into translational and rotational part, considering the inward and outer impacts following up on it [11-20]. In subtle elements, indicating with $\mathrm{m}$ the mass of the aircraft, $\varepsilon=$ (XA , YA , ZA) its position vector concerning the inertial casing GF is the gravitational power, TT the aggregate push, OT the orthogonal change lattice from the body edge to the inertial one, the translational segment peruses: 


$$
\mathrm{m} \varepsilon=\mathrm{GF}+\mathrm{OT} * \mathrm{TT}
$$

The improvement of an appropriate continuous environment for flight control must satisfy a few imperatives keeping in mind the goal to guarantee the convenient preparing of data. The imperatives incorporate the continuous correspondence, the ongoing booking of framework errands, the execution consistency and the counteractive action and the response to basic circumstances. The proposed four rotor aircraft adaptive control algorithm (4R2AC) continuous framework for flight control is described by the engineering appeared in Figure 1and depends on NN.

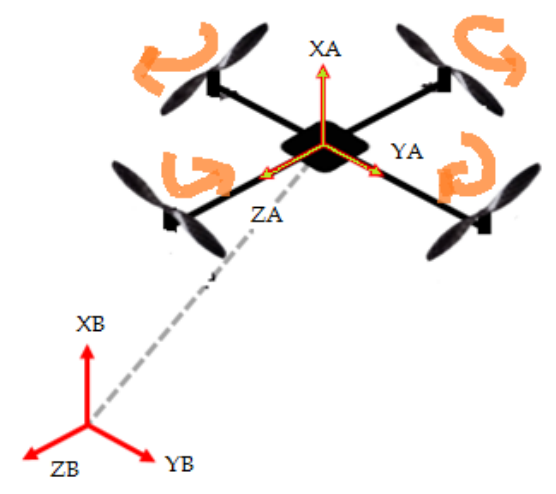

Figure 1. Configuration of Quadruple-Rotor.

Fourfold Rotor, really comprising of four individual settled contribute point cutting edges rotors a " $x$ " or "+" setup. Along these lines the quantity of individual movement degrees of opportunity is six and because of coupled flow, inciting frameworks can't promptly set the increasing velocities in all bearings of space. Because of the four individual movement elements, the Quadruple-Rotor is called an underactuated framework rather than completely impelled. Continuously framework engineering, there is a control framework, in which there is a flight controller alongside the ongoing booking. Inside the flight controller, there exists a constant planning. Quadruple-Rotor is a strong structure with no versatile distortion. Inertial snippet of body in turn is time-invariant, Distribution of the mass of the Quadruple-Rotor is symmetrical which rearrange the conditions, Thrust component of rotors and drag element of Quadruple-Rotor body structure is consistent, Air thickness around is steady. Figure 2 demonstrates the framework engineering of the RT framework.

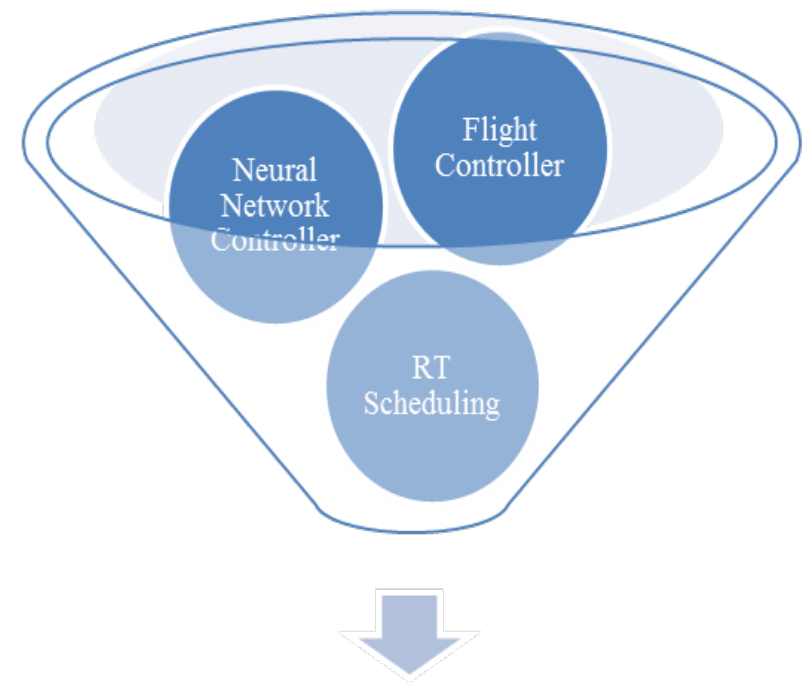

Figure 2. The framework engineering of the RT system.

The NN controler, the flight controller and the RT scheduling framework should be coconsidered in the engineering process. 


\section{Controller of Neural Network}

The continuous framework depends on a NN controller to control the Quadruple-Rotor's movement directions. The algorithm that describes the proposed four rotor aircraft adaptive control algorithm (4R2AC) neural system is appeared in Figure 3.

The neural system controller takes a few setup data as input information, to introduce the constant situating procedure. For example, tables that contain rakish speeds and positions can be such input information's. The neural system controller takes the precise speeds of the four rotors $(\omega 1, \omega 2, \omega 3$ and $(04)$ and after that assesses the evaluated organizes (XA, YA, ZA) as inputs. Also, the outcomes are put away in a database, containing both verifiable of past positions and rakish speeds. Actually, these qualities are utilized for neural system preparing as a part of request to build execution.

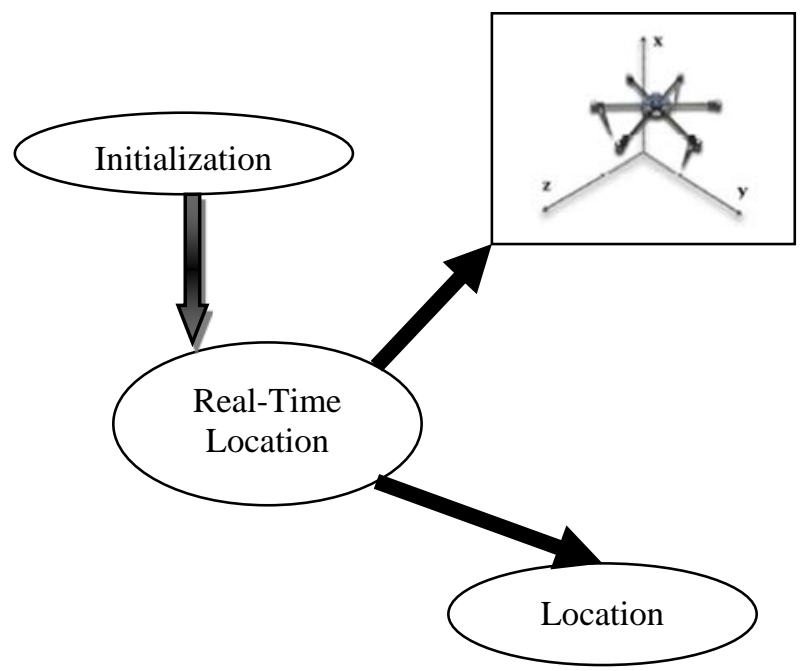

Figure 3. The operation process of controllers in NN.

The indicator connected with the NARX model is displayed concurring the accompanying condition:

The predictor associated with the NARX model is presented according the following equation:

$$
\mathrm{Z}(\mathrm{S} \mid \theta)=\mathrm{Z}(\mathrm{S} \mid \mathrm{S}-1, \theta)=\mathrm{h}(\Phi(\mathrm{S}), \theta)
$$

where $Z$ is the estimation of the variable $Z$ at time $S$ anticipated by the model; $\theta$ is a vector containing the weights of the neural system; $h$ is the capacity acknowledged by the neural system and $\Phi(\mathrm{S})$ is a vector containing the regressors, given by:

$$
+1)] \mathrm{S}
$$

$$
\Phi(S)=[\mathrm{Z}(\mathrm{S}-1) \ldots \mathrm{Z}(\mathrm{S}-\mathrm{na}) \mathrm{v}(\mathrm{S}-\mathrm{nk}) \ldots \mathrm{v}(\mathrm{S}-\mathrm{na}-\mathrm{nk}
$$

where $u$ represents the arrangement of inputs and na, nb and nk are the parameters characterizing the request of the regressors.

The proposed four rotor aircraft adaptive control algorithm (4R2AC) neural system is formed by 20 shrouded neurons, number of postponements equivalent to 2, and utilizes a symmetric sigmoid exchange capacity. Besides, the system is made and prepared in open circle frame. The early ceasing method has been utilized as a part of request to ensure accurate speculation exhibitions to the model and to minimize the danger of over-fitting the preparation information. The whole accessible dataset is part in three subsets: a preparation set, an approval set and a test set. The preparation information set is utilized for figuring the angle of the cost capacity, which is a component of the MSE, and redesigning the system weights. The mistake on the approval set is observed which initiates the preparation procedure. In the proposed four rotor aircraft fuzzy-neural-network-based adaptive control algorithm (4R2AC) NN, the preparation set involves half of the information, while $25 \%$ of the information is utilized as acceptance set and the staying $25 \%$ is held as test set. The anticipating exhibitions of the systems will be evaluated utilizing the test set information and against the 
measured blunder. The real perception is indicated by $\mathrm{s}$ and the gauge is meant by $\mathrm{S}$. by having this gauge mistake is computed at $\mathrm{j}^{\text {th }}$ perception as:

$$
\varepsilon=s j-S j
$$

\section{Simulation}

The height and the disposition control are considered. The positions are kept in control. From the scientific model, the Quadruple-Rotor is a nonlinear, multivariate and emphatically coupled underactivated control framework. What's more, the framework show additionally vulnerabilities of inertial minutes. General BS control algorithm performs not extremely well in nearness of parameter instability and unsettling influences in blustery situations. In figure 4 to figure 6, ZA, YA and XA sights of the pursuing faulty signal is shown, respectively. Figure 7 to figure 9 exhibits the Control Effort at Altitude $\kappa 1, \kappa 2$ and $\kappa 3$.

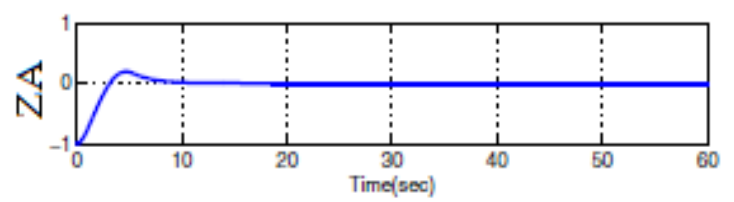

Figure 4. ZA sight of pursuing fault signal

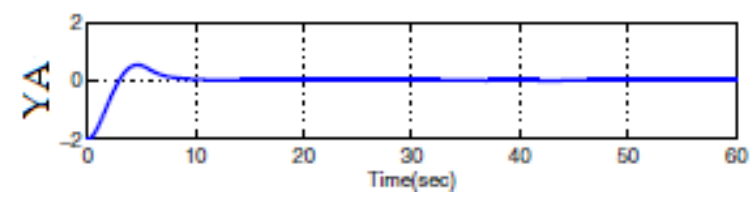

Figure 5. YA sight of pursuing fault signal

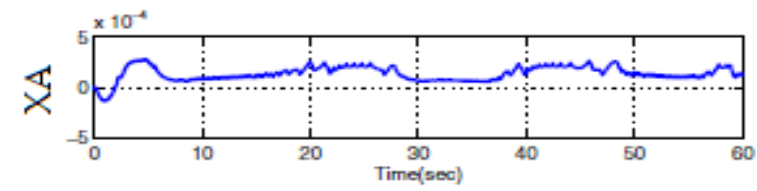

Figure 6. XA sight of pursuing fault signal

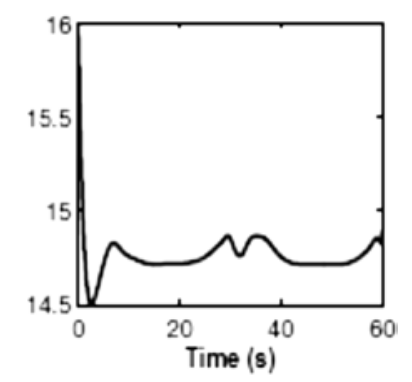

Figure 7. Control Effort at Altitude $\Lambda 1$

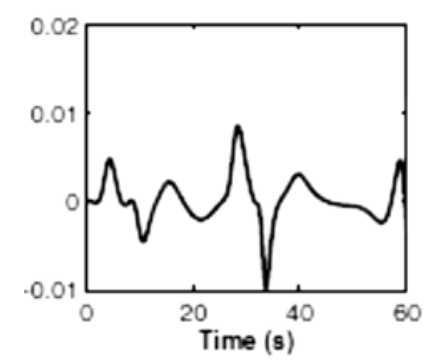

Figure 8. Control Effort at Altitude $\kappa 2$ 


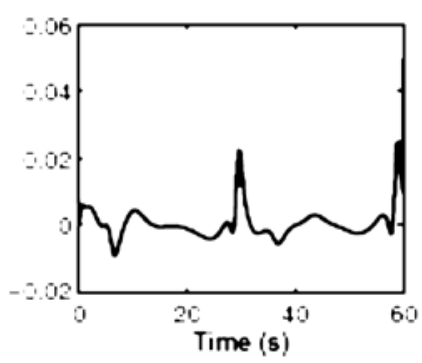

Figure 9. Control Effort at Altitude $K 3$

From figures 4-6, it can be seen that the fault signal is successfully observed and received by the adaptive controller. The controller then quickly responds to the fault signal and generates control outputs which stabilizes the movement of the aircraft. From figure 7-9, it can be seen that control effort at different altitudes are quickly changing with time. The control effort then adjusts the output of the propellers, resulting in adaptive self-control of the flight status of the aircraft.

\section{Conclusion}

In this paper, a direction following control algorithm for four-rotor aircrafts' flight status based on optimized fuzzy neural network is described. Starting from description of a dynamic nonlinear model of quadruple-rotor, the diverse material science wonders are taken into consideration which can impact the advancement of our framework. The proposed four rotor aircraft adaptive control algorithm (4R2AC) control laws lead to a negative semi-unequivocal time subordinate capacity of shut circle framework. Additionally, the underlying states of Quadruple-Rotor states have been considered while breaking down control execution in direction following. Simulation results show that the adaptive IBS cancels the impact of steady twist and disturbance. As the Quadruple-Rotor is influenced by unknown and unpredictable factors along the way, the adaptive algorithm gauges obscure parameters of body structure as well.

\section{References}

[1] Hamel, T., Mahony, R., Lozano, R., and Ostrowski, J., "Dynamic Modeling and Configuration Stabilization for an X4-flyer,” IFAC 15th Triennial World Congress, Barcelona, Spain, (2002), pp. 2012-2017.

[2] Castillo, P., Dzul, A., and Lozano, R., "Real-time Stabilization and Tracking of a Four-rotor Mini Rotorcraft," IEEE Transactions on Control System Technology, Vol. 12, No. 4 (2004), pp. 510-516.

[3] Bouabdallah, S., and Siegwart, R., "Backstepping and Sliding-mode Techniques Applied to an Indoor Micro Quadrotor,” Proceedings of the 2005 IEEE International Conference on Robotics and Automation, Barcelona, Spain, (2005), pp. 2259-2264.

[4] Tayebi, A., and McGilcary, S., “Attitude Stabilization of a VTOL Quadrotor Aircraft,” IEEE Transactions on Control Systems Technology, Vol. 14, No. 3 (2006), pp. 562-571.

[5] Kendoul, F., Fantoni, I., and Lozano, R., "Asymptotic Stability of Hierarchical Inner-Outer Loop-Based Flight Controllers,” Proceedings of the 17th World Congress of IFAC, (2008) pp. 1741-1746.

[6] Yokoyama. M., and Fujimoto, K., Velocity Tracking Control of a Four-rotor Mini Helicopter, Motion and Vibration Control (2009), pp. 335-344, Springer Heidelberg.

[7] Kendoul, F., Yu, Z., and Nonami, K., "Guidance and Nonlinear Control System for Autonomous Flight of Minirotorcraft Unmanned Aerial Vehicles,” Journal of Field Robotics, Vol. 27, No.3 (2010), pp. 311-334. 
[8] Nonami, K., Kendoul, F., Suzuki, S., Wang, W., and Nakazawa, D., Autonomous Flying Robots-Unmanned Aerial Vehicles and Micro Aeria (2010), Springer Japan.

[9] Benallegue, A., Mokhtari, A., and Fridman, L., "High-order Sliding-mode Observer for a Quadrotor UAV,” International Journal of Robust and Nonlinear Control, Vol. 18, (2008), pp. 427440.

[10] Raffo, G. V., Ortega, M. G., and Rubio F. R., "An Integral Predictive/ Nonlinear Hळ Control Structure for a Quadrotor Helicopter,” Automatica, Vol. 46, (2010), pp. 29-39.

[11] Astolfi, A., and Ortega, R., "Immersion and Invariance a New Tool for Stabilization and Adaptive Control of Nonlinear Systems," IEEE Transactions on Automatic Control, Vol. 48, No.4 (2003), pp. 590-606.

[12] Astolfi, A., Karagiannis, D., and Ortega, R., "Nonlinear and Adaptive Control with Applications,” (2008), p. 58, Springer.

[13] Liu, X., Ortega, R., Su, H., and Chu, J., “On Adaptive Control of Nonlinearly Parameterized Nonlinear Systems: Towards a Constructive Procedure,” System \& control Letters, Vol. 60, (2010), pp36-43.

[14] Lee, W. K., and Singh, N. S., “Noncertainty-Equivalent Adaptive Missile Control via Immersion and Invariance,” Journal fo Guidance, Control, and Dynamics, Vol. 33, No. 3 (2010), pp655-665.

[15] Y. Rochefort, H. Piet-Lahanier, S. Bertrand, D. Beauvois and D. Dumur, "Model predictive control of cooperative vehicles using systematic search approach”, Control Engineering Practice, (2014), pp. 204- 217.

[16] G. Zhang , H.t. Zhang, L. Li, L. Wang, "Design of Quad-rotor Micro Air Vehicle”, Journal of Harbin University of Science and Technology, (2012), pp. 110-114.

[17] L.C. Lai, C.C. Yang and C.J. Wu, "Time-Optimal Control of a Hovering Quad-Rotor Helicopter”, Journal of Intelligent and Robotic Systems, (2006), pp. 115-135.

[18] F.H. Jin and H.P. Wang, “Tracking Algorithm of FFsr Based on Fuzzy Logic”, Journal of Harbin University of Science and Technology, (2013), pp. 98-102.

[19] K.K. Veremeenko and V.M. Savel' ev , "In-flight alignment of a strapdown inertial navigation system of an unmanned aerial vehicle”, Journal of Computer and Systems Sciences International, (2013), pp. 106-116. 